\title{
The Hadamard Product in the Space of Lorch Analytic Mappings
}

\author{
by
}

Luiza A. Moraes and Alex F. Pereira

\begin{abstract}
For a complex Banach algebra $E$, let $\mathrm{H}_{L}(E)$ be the space of all mappings from $E$ into $E$ that are analytic in the sense of Lorch, endowed with the Hadamard product and with the topology of uniform convergence on bounded subsets of $E$. We study the topological and algebraic properties of $\mathrm{H}_{L}(E)$ in connection with the topological and algebraic properties of the underlying space $E$. We also study the algebraic and topological properties of the space of sequences $\left(a_{n}\right)_{n} \subset E$ such that $\lim _{n \rightarrow \infty}\left\|a_{n}\right\|^{1 / n}=0$.
\end{abstract}

2010 Mathematics Subject Classification: Primary 46G20; Secondary 46E50, 46J10, 46J20. Keywords: holomorphic mapping, (L)-analytic mapping, Banach algebra, Fréchet algebra, spectrum.

\section{$\S 1$. Introduction}

The theory of Lorch analytic mappings, a special type of holomorphic mappings, goes back to the 1940's and is a natural extension of the classical concept of analytic function to infinite-dimensional algebras. A considerable portion of the classical theory of analytic functions carries over to such mappings (see [9]). The definition of analytic mapping introduced by Lorch in [9] is more restrictive than the standard definition of holomorphic mappings (see [3] or [12]) since Lorch considered only mappings whose domains and ranges are subsets of a commutative Banach algebra with unit. But it is more natural and, in many senses, richer. For instance, it allows concepts like Laurent series, singularities and meromorphic mappings. With Lorch's work as foundation, Blum [1] extended the theory to include a study

Communicated by H. Okamoto. Received October 10, 2011. Revised February 3, 2012.

L. A. Moraes: Instituto de Matemática, Universidade Federal do Rio de Janeiro, CP 68530, CEP 21945-970 Rio de Janeiro, RJ, Brazil;

e-mail: luiza@im.ufrj.br

A. F. Pereira: Universidade Federal do Rio de Janeiro, Polo Cidade Universitária - Campus UFRJ Macaé, Prédio dos laboratórios de Química e Física, CEP 27930-560 Macaé, RJ Brazil; e-mail: alexpereira@im.ufrj.br 
of Laurent expansions, rational functions, and singularities of analytic functions, and Glickfeld [5] presented a definition of meromorphic mappings and proved a generalization of the Mittag-Leffler Theorem.

In the first part of this paper we study the space $\mathrm{H}_{L}(E)$ of mappings from $E$ into $E$ that are analytic in the sense of Lorch, endowed with the Hadamard product and with the topology $\tau_{b}$ of uniform convergence on bounded subsets of $E$. We show that $\mathrm{H}_{L}(E)$ is a commutative (m-convex) Fréchet algebra without unit whose spectrum $\mathcal{M}\left(\mathrm{H}_{L}(E)\right.$ ) is homeomorphic to $\mathcal{M}(E) \times \mathbb{N}_{0}$ (where $\mathcal{M}(E)$ is the spectrum of $E$ and $\mathbb{N}_{0}=\mathbb{N} \cup\{0\}$ ); as a consequence, we show that the algebra $\mathrm{H}_{L}(E)$ is semisimple whenever $E$ is.

In connection with the study of $\mathrm{H}_{L}(E)$, in the second part of this paper we consider the space

$$
\Gamma(E)=\left\{\left(a_{n}\right)_{n} \subset E ; \lim _{n \rightarrow \infty}\left\|a_{n}\right\|^{1 / n}=0\right\} .
$$

Endowed with the usual operations of addition, scalar multiplication and product in spaces of sequences, $\Gamma(E)$ is a non-unital commutative algebra. We show that this algebra, endowed with the topology associated to the metric

$$
d(a, b)=\sup \left\{\left\|a_{0}-b_{0}\right\| ;\left\|a_{n}-b_{n}\right\|^{1 / n}, n \in \mathbb{N}\right\}
$$

is isomorphic to the algebra $\mathrm{H}_{L}(E)$ (under the Hadamard product) and the isomorphism between $\Gamma(E)$ and $\mathrm{H}_{L}(E)$ is in fact a homeomorphism and preserves the algebra structure. Hence, the study of the algebra $\mathrm{H}_{L}(E)$ leads to a better knowledge of the algebra $\Gamma(E)$.

We recall that if a Fréchet algebra $\mathcal{A}$ does not have a unit we cannot guarantee, in general, that every closed maximal ideal of $\mathcal{A}$ is the kernel of a continuous homomorphism on $\mathcal{A}$. In this paper we show that the latter property does hold for $\mathcal{A}=\mathrm{H}_{L}(E)$, and consequently for $\mathcal{A}=\Gamma(E)$.

\section{§2. Notation, terminology and basic results}

We refer to [3], [4], [12], [8] and [13] for background on holomorphic mappings and on Banach and Fréchet algebras.

If $U$ is an open subset of a commutative Banach algebra $E$ with unit, a mapping $f: U \rightarrow E$ is said to have an $(L)$-derivative $f^{\prime}\left(z_{0}\right) \in E$ at $z_{0} \in U$ if for each $\varepsilon>0$ a $\delta>0$ can be found such that for all $h \in E$ satisfying $\|h\|<\delta$ we have

$$
\left\|f\left(z_{0}+h\right)-f\left(z_{0}\right)-h f^{\prime}\left(z_{0}\right)\right\|<\varepsilon\|h\| .
$$

Moreover, $f$ is said to be $(L)$-analytic in $U$ if it has an $(\mathrm{L})$-derivative at each point of $U$. 
We remark that if $f: U \rightarrow E$ is (L)-analytic in $U$ then it is clearly continuous and Fréchet differentiable in $U$ and hence it is a holomorphic mapping in the usual sense of Definition 5.1 in [12] (see also [3] and [4]). However, it is well known that the converse is not true (cf. [7, p. 116] or [11, Remark 2.3]).

The Taylor series expansion of an (L)-analytic mapping $f$ takes a particularly simple form. More precisely, it is known that $f$ is $(\mathrm{L})$-analytic in $U$ if and only if given any $z_{0} \in U$ there exists a $\rho>0$ and unique elements $a_{n} \in E$ such that $f(z)=\sum_{n=0}^{\infty} a_{n}\left(z-z_{0}\right)^{n}$ for all $z$ in $\left\|z-z_{0}\right\|<\rho$. For details, see for instance [7, Theorems 3.19.1 and 26.4.1]. In the notation of [3] we have $(1 / n !) \widehat{d}^{n} f\left(z_{0}\right)(z)=$ $a_{n} z^{n}$ for every $n \in \mathbb{N}$.

Throughout, unless otherwise stated, $E$ will always be a commutative complex Banach algebra with a unit element $e$ and, without loss of generality, we will suppose that $\|e\|=1$.

For $n \in \mathbb{N}, \mathcal{P}_{L}\left({ }^{n} E\right)$ denotes the space of all (L)-analytic $n$-homogeneous polynomials from $E$ into $E$, and $\mathcal{H}_{L}(E)$ denotes the space of all (L)-analytic mappings $f: E \rightarrow E$. When $n=0$ we define $\mathcal{P}_{L}\left({ }^{0} E\right)$ to be the set of all constant mappings from $E$ into $E$.

We denote by $\mathbb{N}_{0}$ the set $\mathbb{N} \cup\{0\}$. Given any $a \in E$ and $n \in \mathbb{N}_{0}$ we denote by $P_{a, n}$ the polynomial defined by $P_{a, n}(z)=a z^{n}$ for every $z \in E$. As usual $P_{a, 0}(z)=a$ if $n=0$. It is clear that $P_{a, n} \in \mathcal{P}_{L}\left({ }^{n} E\right) \subset \mathcal{H}_{L}(E)$ for all $n \in \mathbb{N}_{0}$. We remark that $P \in \mathcal{P}_{L}\left({ }^{n} E\right)$ if and only if $P=P_{a, n}$ for some $a \in E$.

In [11] we showed that the space $\mathcal{P}_{L}\left({ }^{n} E\right)$ with the usual norm given by $\|P\|_{B_{E}}=\sup _{x \in B_{E}}\|P(x)\|$ is isometrically isomorphic to $E$ and that $\mathcal{H}_{L}(E)$ is a closed subspace of the Fréchet space $\mathcal{H}_{b}(E ; E)$ of holomorphic mappings $f: E \rightarrow E$ that are bounded on bounded subsets of $E$ (endowed with the topology $\tau_{b}$ of uniform convergence on bounded subsets of $E)$. We denote by $\mathcal{H}_{L}(E)$ the Fréchet space $\left(\mathcal{H}_{L}(E), \tau_{b}\right)$. As a consequence of $\mathcal{H}_{L}(E) \subset \mathcal{H}_{b}(E ; E)$ and from the Taylor representation of (L)-analytic mappings we find that $f \in \mathcal{H}_{L}(E)$ if and only if there exists $\left(a_{n}\right)_{n} \subset E$ satisfying $\lim _{n \rightarrow \infty}\left\|a_{n}\right\|^{1 / n}=0$ such that $f=\sum_{n=0}^{\infty} P_{a_{n}, n}$ in $\left(\mathcal{H}_{L}(E), \tau_{b}\right)$. We denote by $\Gamma(E)$ the set of all sequences $\left(a_{n}\right)_{n} \subset E$ satisfying $\lim _{n \rightarrow \infty}\left\|a_{n}\right\|^{\frac{1}{n}}=0$.

\section{$\S 3$. The Hadamard product}

In [11] we studied some natural algebras of (L)-analytic mappings. In particular, we showed that the Fréchet space $\mathcal{H}_{L}(E)$ endowed with the pointwise product becomes a commutative Fréchet algebra that we also denoted by $\mathcal{H}_{L}(E)$ and studied its spectrum. As a consequence, we proved that $\mathcal{H}_{L}(E)$ is semisimple if and only if $E$ is. 
In this paper we are going to consider a different algebraic structure in $\mathcal{H}_{L}(E)$. If $f, g \in \mathcal{H}_{L}(E)$, there exist $\left(a_{n}\right)_{n},\left(b_{n}\right)_{n} \in \Gamma(E)$ such that $f(w)=\sum_{n=0}^{\infty} a_{n} w^{n}$ and $g(w)=\sum_{n=0}^{\infty} b_{n} w^{n}$ for every $w \in E$. It is easy to check that $\left(a_{n} b_{n}\right)_{n} \in \Gamma(E)$ and hence if we define $(f \cdot g)(w)=\sum_{n=0}^{\infty} a_{n} b_{n} w^{n}$ for every $w \in E$ we have $f \cdot g \in \mathcal{H}_{L}(E)$. We will refer to this product as the Hadamard product. It is easy to see that $\mathcal{H}_{L}(E)$ endowed with this product is a commutative algebra without identity. From now on this algebra endowed with the topology $\tau_{b}$ will be denoted by $\mathrm{H}_{L}(E)$ and our aim here is to study its spectrum.

It is known that if $X$ and $Y$ are Banach spaces, the family of seminorms defined by

$$
p_{r}(f)=\sum_{n=0}^{\infty}\left\|\frac{\widehat{d}^{n} f(0)}{n !}\right\| r^{n}
$$

for every $f=\sum_{n=0}^{\infty} P_{n} \in \mathcal{H}_{b}(X ; Y)$ and for every $r \in \mathbb{N}$ generates the topology $\tau_{b}$. In particular, $p_{r}(f)=\sum_{n=0}^{\infty}\left\|a_{n}\right\| r^{n}$ for every $f=\sum_{n=0}^{\infty} P_{a_{n}, n} \in \mathrm{H}_{L}(E) \subset$ $\mathcal{H}_{b}(E ; E)$ and it is easy to verify that $p_{r}(f \cdot g) \leq p_{r}(f) p_{r}(g)$ for every $f, g \in \mathrm{H}_{L}(E)$ and for every $r \in \mathbb{N}$. So, $\mathrm{H}_{L}(E)$ is an (m-convex) Fréchet algebra.

Theorem 3.1. Let $E$ be a commutative Banach algebra with unit e. The mapping

$$
\mathfrak{T}: \mathcal{M}(E) \times \mathbb{N}_{0} \rightarrow \mathcal{M}\left(\mathrm{H}_{L}(E)\right)
$$

defined by $\mathfrak{T}(\varphi, m)(f)=\varphi\left(a_{m}\right)$ for every $f=\sum_{n=0}^{\infty} P_{a_{n}, n} \in \mathrm{H}_{L}(E)$ is bijective.

Proof. Fix $(\varphi, m) \in \mathcal{M}(E) \times \mathbb{N}_{0}$. Clearly $\mathfrak{T}(\varphi, m)$ is an algebra homomorphism and $\mathfrak{T}(\varphi, m) \neq 0$ since $\mathfrak{T}(\varphi, m)\left(P_{w_{0}, m}\right)=\varphi\left(w_{0}\right) \neq 0$ for some $w_{0} \in E$. Moreover, given a bounded set $B \subset \mathrm{H}_{L}(E)$ there exists $\lambda>0$ such that $\sup _{f \in B} \sup _{\|w\| \leq 1}\|f(w)\|$ $<\lambda$ and by using the Cauchy inequality we see that

$$
\left\|a_{m}\right\|=\left\|P_{a_{m}, m}\right\| \leq \sup _{\|w\| \leq 1}\|f(w)\|<\lambda
$$

for all $f=\sum_{n=0}^{\infty} P_{a_{n}, n} \in B$. Thus $\mathfrak{T}(\varphi, m)$ is bounded on bounded subsets of $\mathrm{H}_{L}(E)$, which means that $\mathfrak{T}(\varphi, m)$ is continuous, as $\left(\mathrm{H}_{L}(E), \tau_{b}\right)$ is a bornological space. We have just proved that $\mathfrak{T}(\varphi, m) \in \mathcal{M}\left(\mathrm{H}_{L}(E)\right)$.

Now, let $(\varphi, m),(\psi, n) \in \mathcal{M}(E) \times \mathbb{N}_{0}$ be such that $\mathfrak{T}(\varphi, m)=\mathfrak{T}(\psi, n)$. For each $a \in E$,

$$
\varphi(a)=\mathfrak{T}(\varphi, m)\left(P_{a, m}+P_{a, n}\right)=\mathfrak{T}(\psi, n)\left(P_{a, m}+P_{a, n}\right)=\psi(a)
$$

and so $\varphi=\psi$. Now, if $m \neq n$, it is enough to choose $b \in E$ so that $\varphi(b) \neq 0$ in order to get

$$
\mathfrak{T}(\varphi, m)\left(P_{b, n}\right)=\varphi(0)=0 \neq \varphi(b)=\mathfrak{T}(\varphi, n)\left(P_{b, n}\right),
$$

a contradiction. So, we must have $m=n$ and thus $\mathfrak{T}$ is injective. 
Finally we show that $\mathfrak{T}$ is onto. First of all, to each $\phi \in\left(\mathrm{H}_{L}(E), \tau_{b}\right)^{\prime}$ we can associate a sequence $\left(\phi_{n}\right)_{n}$ of continuous linear functionals on $E$ defined by $\phi_{n}(a)=\phi\left(P_{a, n}\right)$ for all $a \in E$. This sequence clearly satisfies

(a) $\phi(f)=\sum_{n=0}^{\infty} \phi_{n}\left(a_{n}\right)$ for every $f=\sum_{n=0}^{\infty} P_{a_{n}, n} \in\left(\mathrm{H}_{L}(E), \tau_{b}\right)$,

(b) $\left(\left\|\phi_{n}\right\|^{1 / n}\right)_{n}$ is bounded.

Let $\phi \in \mathcal{M}\left(\mathrm{H}_{L}(E)\right)$. There exists $m \in \mathbb{N}_{0}$ such that $\phi_{m}$ is a non-null homomorphism on $E$ since $\phi$ is a non-null homomorphism on $\mathrm{H}_{L}(E)$. Now suppose that there exist $m_{1}, m_{2} \in \mathbb{N}_{0}$ such that $m_{1} \neq m_{2}$ and $\phi_{m_{1}}, \phi_{m_{2}} \in \mathcal{M}(E)$. From

$$
\left(P_{e, m_{1}}+P_{e, m_{2}}\right)^{2}=P_{e, m_{1}}+P_{e, m_{2}} \quad \text { and } \quad \phi_{m_{1}}(e)=\phi_{m_{2}}(e)=1
$$

it follows that

$$
\begin{aligned}
4 & =\left(\phi_{m_{1}}(e)+\phi_{m_{2}}(e)\right)^{2}=\left(\phi\left(P_{e, m_{1}}\right)+\phi\left(P_{e, m_{2}}\right)\right)^{2} \\
& =\left(\phi\left(P_{e, m_{1}}+P_{e, m_{2}}\right)\right)^{2}=\phi\left(\left(P_{e, m_{1}}+P_{e, m_{2}}\right)^{2}\right)=\phi\left(P_{e, m_{1}}+P_{e, m_{2}}\right) \\
& =\phi\left(P_{e, m_{1}}\right)+\phi\left(P_{e, m_{2}}\right)=\phi_{m_{1}}(e)+\phi_{m_{2}}(e)=2,
\end{aligned}
$$

absurd. We have just shown the existence of a unique $m \in \mathbb{N}_{0}$ such that $\phi_{m} \neq 0$ and $\phi_{n}=0$ for every $n \neq m$ and hence $\phi(f)=\sum_{n=0}^{\infty} \phi_{n}\left(a_{n}\right)=\phi_{m}\left(a_{m}\right)$ for every $f=\sum_{n=0}^{\infty} P_{a_{n}, n} \in \mathrm{H}_{L}(E)$. Thus, there exists $(\varphi, m) \in \mathcal{M}(E) \times \mathbb{N}_{0}$ satisfying $\phi(f)=\mathfrak{T}(\varphi, m)(f)$ for every $f \in \mathrm{H}_{L}(E)$.

Next we are going to study the continuity of the mapping $\mathfrak{T}$ defined in Theorem 3.1 when $\mathcal{M}\left(\mathrm{H}_{L}(E)\right)$ is endowed with the Gelfand topology $\tau_{G}$ (i.e., the weak* topology), and $\mathbb{N}_{0}$ with the discrete topology.

Theorem 3.2. The spectrum $\mathcal{M}\left(\mathrm{H}_{L}(E)\right)$ is homeomorphic to $\mathcal{M}(E) \times \mathbb{N}_{0}$.

Proof. Let $\mathfrak{T}$ be the bijection between $\mathcal{M}(E) \times \mathbb{N}_{0}$ and $\mathcal{M}\left(\mathrm{H}_{L}(E)\right)$ defined in Theorem 3.1. Take a net $\left(\left(\varphi_{\alpha}, n_{\alpha}\right)\right)_{\alpha \in I}$ in $\mathcal{M}\left(\mathrm{H}_{L}(E)\right) \times \mathbb{N}_{0}$ such that

$$
\begin{array}{ll}
\varphi_{\alpha} \stackrel{\tau_{G}}{\rightarrow} \varphi & \text { in } \mathcal{M}\left(\mathrm{H}_{L}(E)\right), \\
n_{\alpha} \rightarrow m & \text { in } \mathbb{N}_{0} .
\end{array}
$$

By (3.2) there exists $\alpha_{1} \in I$ such that $n_{\alpha}=m$ for all $\alpha \geq \alpha_{1}$ and so, given $f=\sum_{n=0}^{\infty} P_{a_{n}, n} \in \mathrm{H}_{L}(E)$, we have $\mathfrak{T}\left(\varphi_{\alpha}, n_{\alpha}\right)(f)=\varphi_{\alpha}\left(a_{m}\right)$ for every $\alpha \geq \alpha_{1}$. As $a_{m} \in E$, by (3.1) we get $\varphi_{\alpha}\left(a_{m}\right) \rightarrow \varphi\left(a_{m}\right)$ and hence, given $\epsilon>0$, there exists $\alpha_{2} \in I$ such that $\left|\varphi_{\alpha}\left(a_{m}\right)-\varphi\left(a_{m}\right)\right|<\epsilon$ for every $\alpha \geq \alpha_{2}$. Now, if we take any $\alpha_{0} \in I$ satisfying $\alpha_{0} \geq \alpha_{1}$ and $\alpha_{0} \geq \alpha_{2}$ we have

$$
\left|\mathfrak{T}\left(\varphi_{\alpha}, n_{\alpha}\right)(f)-\mathfrak{T}(\varphi, m)(f)\right|=\left|\varphi_{\alpha}\left(a_{m}\right)-\varphi\left(a_{m}\right)\right|<\epsilon \quad \text { for every } \alpha \geq \alpha_{0},
$$


and this is true for every $f \in \mathrm{H}_{L}(E)$. So, $\mathfrak{T}\left(\varphi_{\alpha}, n_{\alpha}\right) \stackrel{\tau_{G}}{\longrightarrow} \mathfrak{T}(\varphi, m)$ in $\mathcal{M}\left(\mathrm{H}_{L}(E)\right)$, showing that $\mathfrak{T}$ is continuous.

It remains to show the continuity of the inverse of $\mathfrak{T}$. Let $\left(\varphi_{\alpha}\right)_{\alpha \in I}$ and $\left(n_{\alpha}\right)_{\alpha \in I}$ be nets in $\mathcal{M}(E)$ and $\mathbb{N}_{0}$, respectively, and let $(\varphi, m) \in \mathcal{M}(E) \times \mathbb{N}_{0}$ be such that

$$
\mathfrak{T}\left(\varphi_{\alpha}, n_{\alpha}\right) \stackrel{\tau_{G}}{\longrightarrow} \mathfrak{T}(\varphi, m) \quad \text { in } \mathcal{M}\left(\mathrm{H}_{L}(E)\right) .
$$

Suppose that $\left(\varphi_{\alpha}, n_{\alpha}\right) \nrightarrow(\varphi, m)$ in $\mathcal{M}(E) \times \mathbb{N}_{0}$ endowed with the product topology. If for every $\alpha \in I$ there exists $\beta_{\alpha} \geq \alpha$ such that $n_{\beta_{\alpha}} \neq m$, then for each $a \in E$ we have $\mathfrak{T}\left(\varphi_{\beta_{\alpha}}, n_{\beta_{\alpha}}\right)\left(P_{a, m}\right)=\varphi_{\beta_{\alpha}}(0)=0$ for every such $\beta_{\alpha}$. But by (3.3) there exists $\alpha_{0} \in I$ satisfying

$$
\left|\mathfrak{T}\left(\varphi_{\alpha}, n_{\alpha}\right)\left(P_{a, m}\right)-\mathfrak{T}(\varphi, m)\left(P_{a, m}\right)\right|<\epsilon \quad \text { for all } \alpha \geq \alpha_{0},
$$

and so, in particular, if we take $\beta_{0} \geq \alpha_{0}$ such that $n_{\beta_{0}} \neq m$ we get $\mathfrak{T}\left(\varphi_{\beta_{0}}, n_{\beta_{0}}\right)\left(P_{a, m}\right)$ $=0$ and $\left|\mathfrak{T}\left(\varphi_{\beta_{0}}, n_{\beta_{0}}\right)\left(P_{a, m}\right)-\varphi(a)\right|<\epsilon$. Thus, $\varphi(a)=0$ for every $a \in E$, a contradiction. Hence, $n_{\alpha} \rightarrow m$ in $\mathbb{N}_{0}$. Now, take $\alpha_{1} \in I$ such that $n_{\alpha}=m$ for every $\alpha \geq \alpha_{1}$. Clearly, for each $a \in E$ we have $\mathfrak{T}\left(\varphi_{\alpha}, n_{\alpha}\right)\left(P_{a, m}\right)=\varphi_{\alpha}(a)$ for every $\alpha \geq \alpha_{1}$. Moreover, given $\epsilon>0$, by (3.3) there exists $\alpha_{2} \in I$ such that $\left|\mathfrak{T}\left(\varphi_{\alpha}, n_{\alpha}\right)\left(P_{a, m}\right)-\mathfrak{T}(\varphi, m)\left(P_{a, m}\right)\right|<\epsilon$ for all $\alpha \geq \alpha_{2}$, and if we choose $\alpha_{0} \in I$ such that $\alpha_{0} \geq \alpha_{1}$ and $\alpha_{0} \geq \alpha_{2}$ we get

$$
\left|\varphi_{\alpha}(a)-\varphi(a)\right|=\left|\mathfrak{T}\left(\varphi_{\alpha}, n_{\alpha}\right)\left(P_{a, m}\right)-\mathfrak{T}(\varphi, m)\left(P_{a . m}\right)\right|<\epsilon
$$

for every $\alpha \geq \alpha_{0}$. From this we infer $\varphi_{\alpha} \stackrel{\tau_{G}}{\longrightarrow} \varphi$ in $\mathcal{M}(E)$, which completes the proof of the continuity of the inverse of $\mathfrak{T}$.

It is well known that if $\mathcal{A}$ is a commutative Fréchet algebra with unit, then the correspondence that to each $\phi \in \mathcal{M}(\mathcal{A})$ associates $\phi^{-1}(0)$ establishes a bijection between $\mathcal{M}(\mathcal{A})$ and the set of all closed maximal ideals of $\mathcal{A}$ (see, for instance, [6, p. 82]). But if $\mathcal{A}$ is a commutative Fréchet algebra without unit we cannot guarantee, in general, that every closed maximal ideal of $\mathcal{A}$ is the kernel of a continuous homomorphism on $\mathcal{A}$. Next we show that $\mathcal{A}=\mathrm{H}_{L}(E)$ does have this property.

Proposition 3.3. If $\mathcal{I}$ is a closed maximal ideal in $\mathrm{H}_{L}(E)$, then there exists $\phi \in \mathcal{M}\left(\mathrm{H}_{L}(E)\right)$ such that $\mathcal{I}=\phi^{-1}(0)$.

Proof. Let $\mathcal{I}$ be a closed maximal ideal in $\mathrm{H}_{L}(E)$. For each $k \in \mathbb{N}_{0}$ define $\pi_{k}$ : $\mathrm{H}_{L}(E) \rightarrow E$ by $\pi_{k}(f)=a_{k}$ for all $f=\sum_{n=0}^{\infty} P_{a_{n}, n} \in \mathrm{H}_{L}(E)$ and let $\mathcal{I}_{k}=\pi_{k}(\mathcal{I})$. It is easy to verify that $\pi_{k}$ is a surjective homomorphism and that $\mathcal{I}_{k}$ is an ideal in $E$. 
We claim that there exists $m \in \mathbb{N}_{0}$ such that $\mathcal{I}_{m} \subsetneq E$. Indeed, suppose that $\mathcal{I}_{k}=E$ for every $k \in \mathbb{N}_{0}$, take any $f=\sum_{n=0}^{\infty} P_{a_{n}, n} \in \mathrm{H}_{L}(E)$ and fix $k \in \mathbb{N}_{0}$ arbitrary. As $E=\pi_{k}(\mathcal{I})$ there exists $g \in \mathcal{I}$ such that $\pi_{k}(g)=\pi_{k}(f)$. Clearly $g \cdot P_{e, k} \in \mathcal{I}$. Moreover, from $\pi_{n}\left(g \cdot P_{e, k}\right)=0$ if $n \neq k$ and $\pi_{k}\left(g \cdot P_{e, k}\right)=\pi_{k}(f)$ we get $P_{a_{k}, k}=g \cdot P_{e, k} \in \mathcal{I}$. Thus, $P_{a_{k}, k} \in \mathcal{I}$ for every $k \in \mathbb{N}_{0}$. As $\mathcal{I}$ is an ideal in $\mathrm{H}_{L}(E)$ we infer that $\sum_{n=0}^{j} P_{a_{n}, n} \in \mathcal{I}$ for every $j \in \mathbb{N}_{0}$. Consequently, $f \in \mathcal{I}$ as $\sum_{n=0}^{j} P_{a_{n}, n} \stackrel{\tau_{b}}{\longrightarrow} f$ and by hypothesis $\mathcal{I}$ is closed. We have just shown that $\mathcal{I}_{k}=E$ for every $k \in \mathbb{N}_{0}$ implies $\mathcal{I}=\mathrm{H}_{L}(E)$, and this contradicts the hypothesis that $\mathcal{I}$ is a maximal ideal in $\mathrm{H}_{L}(E)$. So, the claim is true.

Next we show that $\mathcal{I}_{m}$ is a maximal ideal in $E$. Indeed, let $\mathcal{K}_{m}$ be an ideal in $E$ such that $\mathcal{I}_{m} \subsetneq \mathcal{K}_{m}$. Clearly $\mathcal{K}=\pi_{m}^{-1}\left(\mathcal{K}_{m}\right)$ is an ideal in $\mathrm{H}_{L}(E)$. Also by hypothesis there exists $w \in \mathcal{K}_{m}$ such that $w \notin \mathcal{I}_{m}$, and since $\pi_{m}$ is onto $E$ we may choose $g \in \mathrm{H}_{L}(E)$ such that $\pi_{m}(g)=w \in \mathcal{K}_{m}$. So, $g \in \mathcal{K}$. But $w \notin \mathcal{I}_{m}$ implies $g \notin \mathcal{I}$ and hence $\mathcal{K}$ is an ideal in $\mathrm{H}_{L}(E)$ such that $\mathcal{I} \subsetneq \mathcal{K}$. Since $\mathcal{I}$ is a maximal ideal in $\mathrm{H}_{L}(E)$ this implies $\mathcal{K}=\mathrm{H}_{L}(E)$ and so $\mathcal{K}_{m}=\pi_{m}(\mathcal{K})=\pi_{m}\left(\mathrm{H}_{L}(E)\right)=E$. Thus $\mathcal{I}_{m}$ is a maximal ideal in $E$.

Next we will prove that $\mathcal{I}=\pi_{m}^{-1}\left(\mathcal{I}_{m}\right)$. Clearly $\pi_{m}^{-1}\left(\mathcal{I}_{m}\right) \supset \mathcal{I}$ and $\pi_{m}^{-1}\left(\mathcal{I}_{m}\right)$ is an ideal in $\mathrm{H}_{L}(E)$. But $\mathcal{I}_{m}$ is a maximal ideal in $E$ and so there exists $w \in E$ such that $w \notin \mathcal{I}_{m}$; and since $\pi_{m}$ is onto there exists $g \in \mathrm{H}_{L}(E)$ such that $\pi_{m}(g)=w$. Hence, $\mathcal{I} \subset \pi_{m}^{-1}\left(\mathcal{I}_{m}\right) \subsetneq \mathrm{H}_{L}(E)$ and as $\mathcal{I}$ is a maximal ideal in $\mathrm{H}_{L}(E)$ we get

$$
\mathcal{I}=\pi_{m}^{-1}\left(\mathcal{I}_{m}\right)=\left\{f \in \mathrm{H}_{L}(E) ; \pi_{m}(f) \in \mathcal{I}_{m}\right\} .
$$

Finally, since $\mathcal{I}_{m}$ is a maximal ideal in the Banach algebra (commutative with unit) $E$, there exists $\varphi \in \mathcal{M}(E)$ such that $\mathcal{I}_{m}=\varphi^{-1}(0)$. Hence

$$
\mathcal{I}=\left\{f=\sum_{n=0}^{\infty} P_{a_{n}, n} \in \mathrm{H}_{L}(E) ; a_{m} \in \varphi^{-1}(0)\right\} .
$$

So, by Theorem 3.1 there exists $\phi:=\mathfrak{T}(\varphi, m) \in \mathcal{M}\left(\mathrm{H}_{L}(E)\right)$ such that

$$
\mathcal{I}=\left\{f \in \mathrm{H}_{L}(E) ; \phi(f)=0\right\}=\phi^{-1}(0) .
$$

We recall that the $\operatorname{radical} \mathcal{R}(\mathcal{A})$ of a commutative algebra $\mathcal{A}$ is the intersection of all maximal ideals in $\mathcal{A}$. An algebra $\mathcal{A}$ is called semisimple if $\mathcal{R}(\mathcal{A})=\{0\}$. As $\mathrm{H}_{L}(E)$ is a commutative (Fréchet) algebra, the kernel of any non-null continuous homomorphism from $\mathrm{H}_{L}(E)$ into $\mathbb{C}$ is a closed maximal ideal in $\mathrm{H}_{L}(E)$ (use Lemma 7.1 p. 68 of [10] and continuity) and we have

$$
\mathcal{R}\left(\mathrm{H}_{L}(E)\right) \subset \bigcap_{\phi \in \mathcal{M}\left(\mathrm{H}_{L}(E)\right)} \phi^{-1}(0) .
$$

The next proposition follows from Theorem 3.1. 
Proposition 3.4. The algebra $\mathrm{H}_{L}(E)$ is semisimple whenever $E$ is.

Proof. Given $\varphi \in \mathcal{M}(E)$ and $m \in \mathbb{N}_{0}$, let $\phi=\mathfrak{T}(\varphi, m)$ where $\mathfrak{T}$ is defined as in Theorem 3.1. Clearly for each $f=\sum_{n=0}^{\infty} P_{a_{n}, n} \in \mathrm{H}_{L}(E)$ we know that $\phi(f)=0$ if and only if $\varphi\left(a_{m}\right)=0$. So, the set

$$
X_{\varphi, m}=\left\{f=\sum_{n=0}^{\infty} P_{a_{n}, n} \in \mathrm{H}_{L}(E) ; a_{m} \in \varphi^{-1}(0)\right\}
$$

is a closed maximal ideal in $\mathrm{H}_{L}(E)$ since $X_{\varphi, m}=\phi^{-1}(0)$ and $\phi \in \mathcal{M}\left(\mathrm{H}_{L}(E)\right)$. Now, by using the fact that $\mathcal{R}\left(\mathrm{H}_{L}(E)\right) \subset \bigcap_{\phi \in \mathcal{M}\left(\mathrm{H}_{L}(E)\right)} \phi^{-1}(0)$ and Theorem 3.1 we deduce that $f \in X_{\varphi, m}$ for all $\varphi \in \mathcal{M}(E)$ and $m \in \mathbb{N}_{0}$ whenever $f$ is in $\mathcal{R}\left(\mathcal{H}_{L}(E)\right)$. Consequently, given any $f=\sum_{n=0}^{\infty} P_{a_{n}, n} \in \mathcal{R}\left(\mathrm{H}_{L}(E)\right)$ we see that $a_{n} \in \bigcap_{\varphi \in \mathcal{M}(E)} \varphi^{-1}(0)=\mathcal{R}(E)$ for every $n \in \mathbb{N}_{0}$. Hence $a_{n}=0$ for all $n \in \mathbb{N}_{0}$ since, by hypothesis, $\mathcal{R}(E)=0$. This shows that $f \equiv 0$ for every $f \in \mathcal{R}\left(\mathcal{H}_{L}(E)\right)$, and consequently $\mathrm{H}_{L}(E)$ is semisimple.

\section{$\S 4$. The sequence space $\Gamma(E)$}

Let $\Gamma(E)=\left\{\left(a_{n}\right)_{n} \subset E ; \lim _{n \rightarrow \infty}\left\|a_{n}\right\|^{1 / n}=0\right\}$. One checks easily that $\Gamma(E)$ endowed with the usual addition and scalar multiplication operations is a vector space.

We define a topological structure in $\Gamma(E)$ and establish an isomorphism between $\Gamma(E)$ and $\mathrm{H}_{L}(E)$.

Proposition 4.1. The mapping $d: \Gamma(E) \times \Gamma(E) \rightarrow \mathbb{R}$ defined by

$$
d(a, b)=\sup \left\{\left\|a_{0}-b_{0}\right\| ;\left\|a_{n}-b_{n}\right\|^{1 / n}, n \in \mathbb{N}\right\}
$$

for all $a=\left(a_{n}\right)_{n}$ and $b=\left(b_{n}\right)_{n}$ in $\Gamma(E)$ is a translation invariant metric in $\Gamma(E)$.

Proof. For all $a, b \in \Gamma(E)$ it is clear that $0 \leq d(a, b)<\infty, d(a, b)=0$ if and only if $a=b$, and $d(a, b)=d(b, a)$. Moreover, given $a=\left(a_{n}\right)_{n}, b=\left(b_{n}\right)_{n}, c=\left(c_{n}\right)_{n}$ in $\Gamma(E)$, the triangle inequality $d(a, c) \leq d(a, b)+d(b, c)$ follows from the fact that

$$
\left\|a_{n}-c_{n}\right\|^{1 / n} \leq\left\|a_{n}-b_{n}\right\|^{1 / n}+\left\|b_{n}-c_{n}\right\|^{1 / n} \quad \text { for all } n \in \mathbb{N} .
$$

Finally, it is easy to check that $d(a+c, b+c)=d(a, b)$ for all $a, b, c \in \Gamma(E)$.

Remark 4.2. (a) For every $\lambda \in \mathbb{C}$ and $a, b \in \Gamma(E)$,

$$
d(\lambda a, \lambda b) \leq A(\lambda) d(a, b) \quad \text { where } \quad A(\lambda)=\max \{1,|\lambda|\} .
$$

(b) Directly from the definition of $d(x, 0)$ it follows that

$$
d(a+b, 0) \leq d(a, 0)+d(b, 0) \quad \text { for all } a, b \in \Gamma(E) .
$$


Proposition 4.3. $(\Gamma(E), d)$ is a topological vector space.

Proof. Addition is continuous at the origin by Remark 4.2(b) and hence at any other point by translation invariance of $d$.

Let us show that scalar multiplication is also continuous. Since $d$ is translation invariant, all we have to show is that $d\left(\lambda_{p} a^{p}, 0\right) \rightarrow 0$ whenever $\left(a^{p}\right) \subset \Gamma(E)$, $a \in \Gamma(E)$ and $\left(\lambda_{p}\right) \subset \mathbb{C}$ satisfy $d\left(a^{p}, a\right) \rightarrow 0$ and $\lambda_{p} \rightarrow 0$. Without loss of generality we may suppose that $\left|\lambda_{p}\right| \leq 1$ for all $p \in \mathbb{N}$. Let $a^{p}=\left(a_{p, n}\right)_{n}$ for all $p \in \mathbb{N}$ and $a=\left(a_{n}\right)_{n}$. Fix $\epsilon>0$. As $a=\left(a_{n}\right)_{n} \in \Gamma(E)$, there exists $n_{0} \in \mathbb{N}$ such that

$$
\left\|a_{n}\right\|^{1 / n}<\epsilon / 2 \quad \text { for all } n \geq n_{0} .
$$

Moreover, there exists $p_{0} \in \mathbb{N}$ such that for every $p \geq p_{0}$ we have $d\left(a^{p}, a\right)<\epsilon / 2$ and

$$
\left\|\lambda_{p} a_{0}\right\|<\epsilon / 2 \text { and }\left\|\lambda_{p} a_{n}\right\|^{1 / n}<\epsilon / 2 \text { for } n=1, \ldots, n_{0}-1
$$

Also

$$
\left\|a_{p, 0}-a_{0}\right\|<\epsilon / 2 \text { and }\left\|a_{p, n}-a_{n}\right\|^{1 / n}<\epsilon / 2 \quad \text { for all } n \in \mathbb{N} \text {. }
$$

Now, by using (4.2) and (4.3) we get

$$
\left\|\lambda_{p} a_{p, 0}\right\| \leq\left|\lambda_{p}\right|\left\|a_{p, 0}-a_{0}\right\|+\left\|\lambda_{p} a_{0}\right\|<\epsilon
$$

for every $p \geq p_{0}$ and

$$
\left\|\lambda_{p} a_{p, n}\right\|^{1 / n} \leq\left|\lambda_{p}\right|^{1 / n}\left\|a_{p, n}-a_{n}\right\|^{1 / n}+\left\|\lambda_{p} a_{n}\right\|^{1 / n}<\epsilon
$$

for $n=1, \ldots, n_{0}-1$. Moreover, for all $p \geq p_{0}$ and $n \geq n_{0}$, by (4.1) we get

$$
\left\|\lambda_{p} a_{p, n}\right\|^{1 / n} \leq\left|\lambda_{p}\right|^{1 / n}\left\|a_{p, n}-a_{n}\right\|^{1 / n}+\left|\lambda_{p}\right|^{1 / n}\left\|a_{n}\right\|^{1 / n}<\epsilon .
$$

Finally, (4.4)-(4.6) imply $d\left(\lambda_{p} a^{p}, 0\right) \leq \epsilon$ for all $p \geq p_{0}$.

Proposition 4.4. The metric space $(\Gamma(E), d)$ is complete.

Proof. Let $\left(a_{p}\right)_{p}$ be a Cauchy sequence in $\Gamma(E)$. Let $a_{p}=\left(a_{p, n}\right)_{n}$ for all $p \in \mathbb{N}$. Given $0<\epsilon<1$, there exists $r \in \mathbb{N}$ such that

$$
\begin{array}{r}
\left\|a_{p, 0}-a_{q, 0}\right\|<\epsilon, \\
\left\|a_{p, n}-a_{q, n}\right\|^{1 / n}<\epsilon,
\end{array}
$$

for all $n \in \mathbb{N}$ and $p, q \geq r$. It follows that $\left(a_{p, n}\right)_{p}$ is a Cauchy sequence in $E$ for all $n \in \mathbb{N}_{0}$ and so there exists $a_{n} \in E$ such that $a_{p, n} \rightarrow a_{n}$ as $p \rightarrow \infty$, for all $n \in \mathbb{N}_{0}$. 
Fix $n \in \mathbb{N}_{0}$ and $p \geq r$; by letting $q \rightarrow \infty$ in (4.7) and (4.8) we get

$$
\begin{aligned}
\left\|a_{p, 0}-a_{0}\right\| & <\epsilon, \\
\left\|a_{p, n}-a_{n}\right\|^{1 / n} & <\epsilon, \quad n \geq 1,
\end{aligned}
$$

for all $p \geq r$. Moreover $\lim _{n \rightarrow \infty}\left\|a_{p, n}\right\|^{1 / n}=0$ for each $p \in \mathbb{N}$ since $a_{p} \in \Gamma(E)$. Now, by taking $p=r$ in (4.10) the triangle inequality yields $\lim _{n \rightarrow \infty}\left\|a_{n}\right\|^{1 / n}=0$, so $a=\left(a_{n}\right)_{n} \in \Gamma(E)$. Finally, (4.9) and (4.10) imply that $d\left(a_{p}, a\right)<\epsilon$ for all $p \geq r$, which completes the proof.

Remark 4.5. For some authors (see, for instance, Conway [2]) a Fréchet space is a topological vector space $X$ whose topology is defined by a translation invariant metric $d$ such that $(X, d)$ is a complete metric space. Propositions 4.3 and 4.4 tell us that $\Gamma(E)$ is a Fréchet space in this sense. Meanwhile, most authors require a Fréchet space to be a locally convex space. Since balls in $\Gamma(E)$ are not convex, a priori we cannot guarantee that $\Gamma(E)$ is a Fréchet space in this more restricted sense. But the next result shows that $\Gamma(E)$ is indeed locally convex.

Theorem 4.6. $(\Gamma(E), d)$ and $\left(\mathrm{H}_{L}(E), \tau_{b}\right)$ are isomorphic as topological vector spaces.

Proof. Define $T:(\Gamma(E), d) \rightarrow\left(\mathrm{H}_{L}(E), \tau_{b}\right)$ by $T(a)=\sum_{n=0}^{\infty} P_{a_{n}, n}$ for all $a=$ $\left(a_{n}\right)_{n} \in \Gamma(E)$. Clearly $T$ is a linear bijection and so all we have to show is that $T$ is a homeomorphism. We will show that if $a=\left(a_{n}\right)_{n} \in \Gamma(E)$ and $\left(a^{p}\right)_{p} \subset \Gamma(E)$, then

$$
d\left(a^{p}, a\right) \rightarrow 0 \text { as } p \rightarrow \infty \text { if and only if } T\left(a^{p}\right) \stackrel{\tau_{b}}{\longrightarrow} T(a) \text { as } p \rightarrow \infty .
$$

Let $a^{p}=\left(a_{p, n}\right)_{n}$ for each $p \in \mathbb{N}$. Given a bounded subset $B$ of $E$, let $R>1$ be such that $\|w\| \leq R$ for every $w \in B$. To each $\epsilon>0$, we may associate $\eta>0$ satisfying $\eta R<1$ and $\eta\left(1+\frac{R}{1-\eta R}\right) \leq \epsilon$. If $d\left(a^{p}, a\right) \rightarrow 0$, there exists $p_{0} \in \mathbb{N}$ such that for all $p \geq p_{0}$ we have

$$
\left\|a_{p, 0}-a_{0}\right\| \leq \eta \quad \text { and } \quad\left\|a_{p, n}-a_{n}\right\| \leq \eta^{n} \quad \text { for every } n \geq 1 .
$$

Hence, for every $w \in B$ and all $p \geq p_{0}$ we have

$$
\begin{aligned}
\left\|T\left(a^{p}\right)(w)-T(a)(w)\right\| & \leq \sum_{n=0}^{\infty}\left\|a_{p, n}-a_{n}\right\|\|w\|^{n} \leq \eta+\sum_{n=1}^{\infty}(\eta R)^{n} \\
& =\eta\left(1+\frac{R}{1-\eta R}\right) \leq \epsilon,
\end{aligned}
$$

i.e., $T\left(a^{p}\right) \rightarrow T(a)$ uniformly on $B$. As this is true for every bounded subset $B$ of $E$, this means that $T\left(a^{p}\right) \stackrel{\tau_{b}}{\longrightarrow} T(a)$. 
Conversely, suppose that $T\left(a^{p}\right) \stackrel{\tau_{b}}{\longrightarrow} T(a)$. In particular, for $B=\{0\}$ we get $\left\|a_{p, 0}-a_{0}\right\| \rightarrow 0$. Given $\epsilon>0$, let $R \geq 1 / \epsilon$. By hypothesis, there exists $p_{0} \in \mathbb{N}$ such that for every $p \geq p_{0}$ we have

$$
\left\|a_{p, 0}-a_{0}\right\| \leq \epsilon \quad \text { and } \quad\left\|T\left(a^{p}\right)(w)-T(a)(w)\right\| \leq 1 \quad \text { for all }\|w\| \leq R .
$$

For all $n \in \mathbb{N}$ and $p \geq p_{0}$ the Cauchy inequality yields

$$
\begin{aligned}
\left\|a_{p, n}-a_{n}\right\| & =\sup _{\|w\| \leq 1}\left\|a_{p, n} w^{n}-a_{n} w^{n}\right\| \leq \sup _{\|w\| \leq R}\left\|a_{p, n} w^{n}-a_{n} w^{n}\right\| \\
& \leq \frac{1}{R^{n}} \sup _{\|w\| \leq R}\left\|T\left(a^{p}\right)(w)-T(a)(w)\right\| \leq \frac{1}{R^{n}},
\end{aligned}
$$

and so $\left\|a_{p, n}-a_{n}\right\|^{1 / n} \leq 1 / R \leq \epsilon$. Hence, $d\left(a^{p}, a\right) \leq \epsilon$ for every $p \geq p_{0}$.

As $\left(\mathrm{H}_{L}(E), \tau_{b}\right)$ is a locally convex space, from Theorem 4.6 and Proposition 4.4 it follows that $(\Gamma(E), d)$ is a Fréchet space in the restricted sense.

Proposition 4.7. The Fréchet space $(\Gamma(E), d)$ is not normable.

Proof. As $(\Gamma(E), d)$ is a locally convex space, by Kolmogorov's Theorem (see [2, p. 107, Proposition 2.6]), it is enough to prove that $\Gamma(E)$ does not have a bounded neighborhood of zero. Fix $\epsilon>0$ and take $\eta=\epsilon / 4$. For each $\lambda>0$, take $m \in \mathbb{N}$ large enough that $\lambda^{1 / m}<2$. If $a=\left(a_{n}\right)_{n} \in \Gamma(E)$ is defined by $a_{n}=0$ for all $n \neq m$ and $a_{m}=(\epsilon / 2)^{m} e$, it is clear that $a \in U_{\epsilon}(0)=\{a \in \Gamma(E) ; d(a, 0)<\epsilon\}$, and moreover $\left\|a_{m} / \lambda\right\|^{1 / m}>\epsilon / 4=\eta$ since $a \notin \lambda U_{\eta}(0)$. This shows that $U_{\epsilon}(0)$ contains an open set $U_{\eta}(0)=\{a \in \Gamma(E) ; d(a, 0)<\eta\}$ such that $U_{\epsilon}(0) \not \subset \lambda U_{\eta}(0)$ for every $\lambda>0$. Hence, $U_{\epsilon}(0)$ is not bounded and this completes the proof.

If we endow $\Gamma(E)$ with the usual product $\left(a_{n}\right) \cdot\left(b_{n}\right)=\left(a_{n} b_{n}\right)_{n}$, the isomorphism established in Theorem 4.6 preserves the algebra structure. So, $T$ and $T^{-1}$ are homomorphisms of algebras. Hence, $\Gamma(E)$ is a commutative Fréchet algebra without unit when endowed with this product.

Proposition 4.8. The spectrum $\mathcal{M}(\Gamma(E))$ is homeomorphic to $\mathcal{M}(E) \times \mathbb{N}_{0}$.

Proof. Define $S: \mathcal{M}(\Gamma(E)) \rightarrow \mathcal{M}\left(\mathrm{H}_{L}(E)\right)$ by $S(\phi)=\phi \circ T^{-1}$ for every $\phi \in$ $\mathcal{M}(\Gamma(E))$, where $T^{-1}$ is the inverse of the mapping $T$ defined in Theorem 4.6. Clearly $\phi \circ T^{-1}$ is a homomorphism from $\mathrm{H}_{L}(E)$ into $\mathbb{C}$. Moreover, as $\phi \in \mathcal{M}(\Gamma(E))$ and $T^{-1}$ is onto, there exists $f \in \mathrm{H}_{L}(E)$ such that $\phi \circ T^{-1}(f) \neq 0$ and so $S$ is well defined. The injectivity of $S$ follows from the fact that $T^{-1}$ is onto and it is clear that $S$ is onto. So, $S$ establishes a bijection between $\mathcal{M}(\Gamma(E))$ and $\mathcal{M}\left(\mathrm{H}_{L}(E)\right)$. On the other hand, the continuity of $T$ and $T^{-1}$ implies the continuity of $S$ and 
$S^{-1}$, so that $S$ is an isomorphism between $\mathcal{M}(\Gamma(E))$ and $\mathcal{M}\left(\mathrm{H}_{L}(E)\right)$. Now the result follows by using Theorems 3.1 and 3.2 .

Proposition 4.9. If $\mathcal{I}$ is a closed maximal ideal in $\Gamma(E)$, then there exists $\psi$ in $\mathcal{M}(\Gamma(E))$ such that $\mathcal{I}=\psi^{-1}(0)$.

Proof. Let $\mathcal{I}$ be a closed maximal ideal in $\Gamma(E)$. From the fact that the mapping $T$ defined in Theorem 4.6 is an injective homomorphism from $\Gamma(E)$ onto $\mathrm{H}_{L}(E)$, we deduce that $T(\mathcal{I})$ is a closed maximal ideal in $\mathrm{H}_{L}(E)$, and so by Proposition 3.3 there exists $\phi \in \mathcal{M}\left(\mathrm{H}_{L}(E)\right)$ such that $T(\mathcal{I})=\phi^{-1}(0)$. Thus, $\psi=\phi \circ T \in \mathcal{M}(\Gamma(E))$ with $\mathcal{I}=\psi^{-1}(0)$.

Proposition 4.10. The algebra $\Gamma(E)$ is semisimple whenever $E$ is.

\section{Acknowledgements}

The first author was supported in part by CNPq, Brazil, Research Grant 305848/ 2009-7. The second author was supported by CNPq, Brazil, Scholarship.

\section{References}

[1] E. K. Blum, A theory of analytic functions in Banach algebras, Trans. Amer. Math. Soc. 78 (1955), 343-370. Zbl 0064.36401 MR 0069405

[2] J. B. Conway, A course in functional analysis, 2nd ed., Grad. Texts in Math. 96, Springer, New York, 1990. Zbl 0706.46003 MR 1070713

[3] S. Dineen, Complex analysis in locally convex spaces, North-Holland Math. Stud. 57, NorthHolland, Amsterdam, 1981. Zbl 0484.46044 MR 0640093

[4] _ Complex analysis on infinite dimensional spaces, Springer Monogr. Math., Springer, London, 1999. Zbl 1034.46504 MR 1705327

[5] B. W. Glickfeld, Meromorphic functions of elements of a commutative Banach algebra, Trans. Amer. Math. Soc. 151 (1970), 293-307. Zbl 0205.13604 MR 0264398

[6] H. Goldmann, Uniform Fréchet algebras, North-Holland Math. Stud. 162, North-Holland, Amsterdam, 1990. Zbl 0718.46017 MR 1049384

[7] E. Hille and R. S. Phillips, Functional analysis and semi-groups, Colloq. Publ. 31, Amer. Math. Soc., Providence, RI, 1974. Zbl 0392.46001 MR 0423094

[8] R. Larsen, Banach algebras. An introduction, Dekker, New York, $1973 . \quad$ Zbl 0264.46042 MR 0487369

[9] E. R. Lorch, The theory of analytic functions in normed abelian vector rings, Trans. Amer. Math. Soc. 54 (1943), 414-425. Zbl 0060.27202 MR 0009090

[10] A. Mallios, Topological algebras. Selected topics, North-Holland Math. Stud. 124, NorthHolland, Amsterdam, 1986. Zbl 0597.46046 MR 0857807

[11] L. A. Moraes and A. F. Pereira, The spectra of algebras of Lorch analytic mappings, Topology 48 (2009), 91-99. Zbl 1194.46068 MR 2596202

[12] J. Mujica, Complex analysis in Banach spaces, North-Holland Math. Stud. 120, NorthHolland, Amsterdam, 1986. Z Zbl 0586.46040 MR 0842435

[13] E. L. Stout, The theory of uniform algebras, Bogden and Quigley, Tarrytown-on-Hudson, New York, 1971. Zbl 0286.46049 MR 0423083 